\title{
Flora of Singapore precursors, 23: Notes on Ochnaceae in Singapore
}

\author{
I.M. Turner ${ }^{1,2}$ \\ ${ }^{1}$ Singapore Botanical Liaison Officer, \\ Royal Botanic Gardens, Kew, Richmond, \\ Surrey, TW9 3AE, U.K. \\ i.turner@kew.org \\ ${ }^{2}$ Singapore Botanic Gardens, National Parks Board, \\ 1 Cluny Road, 259569 Singapore
}

\begin{abstract}
The native Ochnaceae of Singapore (five species from three genera) are listed with synonymy and typification. An epitype is designated for Euthemis elegantissima Wall. to fix the application of the name in the sense of Brackenridgea hookeri (Planch.) A.Gray. In addition, 17 lectotypes (including 13 at the second step) and three neotypes (two at the second step) are designated here.
\end{abstract}

Keywords. Brackenridgea, Campylospermum, Euthemis

\section{Introduction}

The Ochnaceae are a family of over 500 species of woody plants found across the tropical and subtropical regions of the globe. The native flora of Singapore includes five species of Ochnaceae from three genera. The regional revision of the family by Kanis (1968) has been followed closely by nearly all taxonomic works on Asia-Pacific Ochnaceae for the last half century. In revising the family for the Flora of Singapore, Kanis has again been the main author referred to and generally deferred to. However, as was commonly the case in the period, Kanis was not entirely critical when it came to the typification of the names of taxa. While he did generally cite types and even referred to lectotypes and neotypes, he was often imprecise when more than one type specimen was available. Therefore, many names still lack fully resolved typification. The purpose of this paper is to review the nomenclature and typification of names in Ochnaceae relevant to the Flora of Singapore.

\section{Nomenclatural synopsis}

1. Brackenridgea A.Gray, Char. New Gen. Polynesia 5 (1853) [Proc. Amer. Acad. Arts 3: 51 (1857)]. - Ochna sect. Brackenridgea (A.Gray) Kuntze, Lex. Gen. Phan. 394 (1903). - TYPE: Brackenridgea hookeri (Planch.) A.Gray. 
Pleuroridgea Tiegh., Bull. Mus. Hist. Nat. (Paris) 8: 215 (1902). - TYPE: Pleuroridgea zanguebarica (Oliv.) Tiegh. (= Brackenridgea zanguebarica Oliv.).

Campylopora Tiegh., Bull. Mus. Hist. Nat. (Paris) 8: 547 (1902). - TYPE: Campylopora australiana (F.Muell.) Tiegh. (= Brackenridgea nitida A.Gray subsp. australiana (F.Muell.) Kanis).

Notochnella Tiegh., Bull. Mus. Hist. Nat. (Paris) 8: 549 (1902) - Brackenridgea sect. Notochnella (Tiegh.) Kanis, Blumea 16: 43 (1968). - TYPE: Notochnella fascicularis (Blanco) Tiegh. (= Brackenridgea fascicularis (Blanco) Fern.-Vill.).

1.1 Brackenridgea elegantissima (Wall.) Kanis, Blumea 16: 50 (1968). - Euthemis elegantissima Wall. in Roxburgh, Fl. Ind. 2: 305 (1824). - TYPE: Singapore, September 1822, Wallich s.n. [EIC 2518] (lectotype K-W [K001116334], designated by Kanis, Blumea 16: 51 (1968)). Singapore, Bukit Timah, February 1900, Ridley 10738 (epitype K [K000657990], designated here; isoepitype SING [SING0054195]).

Gomphia hookeri Planch., London J. Bot. 6:3 (1847). - Brackenridgea hookeri (Planch.) A.Gray, Char. New Gen. Polynesia 6 (1853) [Proc. Amer. Acad. Arts 3: 51 (1857)]. - Ouratea hookeri (Planch.) Burkill, Bull. Misc. Inform. Kew 1935: 318 (1935). TYPE: Prince of Wales Island [Penang], Anon. s.n. (holotype K [K000501143]).

Brackenridgea perakensis Tiegh., Ann. Sci. Nat., Bot. sér. 8, 16: 396 (1902). TYPE: [Peninsular Malaysia], Perak, Larut, February 1885, King's Collector 7310 (lectotype P [P01817423], designated by Kanis, Blumea 16: 45 (1968); isolectotypes BO, CAL, E [E00034479], K [K000657991], SING [SING0054196, SING0054197, SING0054198]).

Brackenridgea denticulata Furtado, Gard. Bull. Singapore 19: 183 (1962). - TYPE: Singapore, Bukit Timah, February 1900, Ridley 10738 (holotype SING [SING0054195]; isotypes BM [BM000798710], K [K000657990], L [L0012445]).

Notes. During his visit to Singapore in 1822, Nathaniel Wallich collected material from a juvenile Ochnaceae plant. So impressed was Wallich by its elegant leaf venation, better in his opinion than Roxburgh's favourite, the gaharu tree (Aquilaria malaccensis Lam.), he named the plant Euthemis elegantissima. Wallich had also collected the two species of the genus Euthemis described by William Jack in Singapore, so it is perhaps unsurprising that he put his 'most elegant' plant in the same genus. Kanis (1968) noted that Wallich's only known specimen was actually juvenile material of Brackenridgea and made the combination for it in that genus. However, Kanis was not able to determine to which of the two species of Brackenridgea native to Singapore (B. hookeri and B. palustris) the material belonged. In other words, the type was demonstrably ambiguous (Lendemer, 2020). Therefore, Kanis considered the 
name doubtful, though older than both of the names in use. Recently, Govaerts (1996) accepted Brackenridgea elegantissima in the sense of B. hookeri and this has been followed by at least two publications on the Singapore flora (Wong et al., 2013; Ho et al., 2019). It therefore seems prudent to stabilise the nomenclature of the species by designating an epitype to fix the application of the name Brackenridgea elegantissima. A specimen collected by Ridley at Bukit Timah is selected as epitype here. While the only known original material of Euthemis elegantissima is the sheet in the East India Company Herbarium (K-W), it cannot be established that Wallich based his description solely on this specimen. I therefore consider Kanis to have effectively designated the specimen as lectotype, rather than taking it as a holotype.

1.2 Brackenridgea palustris Bartell., Malpighia 15: 165 (1901). - TYPE: Borneo, Danau Lamadjan, Beccari P.B. 3472 (holotype FI [FI007762]; isotypes A [A00898753], K [K000657989], L [L0012450], P [P01817429]).

Brackenridgea hookeri (Planch.) A.Gray var. leucocarpa Scheff., Natuurw. Tijdschr. Ned.-Indië 32: 411 (1873). - TYPE: Banka, Djabus [Djeboes in protologue], Teijsmann s.n. (lectotype BO n.v., designated by Kanis, Blumea 16: 46 (1968); isolectotype L [L0012449]).

Gomphia hookeri Planch. var. corymbosa King, J. Asiat. Soc. Bengal, Pt. 2, Nat. Hist. 62(4): 233 (1894). - Brackenridgea corymbosa (King) Tiegh., Ann. Sci. Nat., Bot. sér. 8, 16: 396 (1902). - Gomphia corymbosa (King) Ridl., J. Straits Branch Roy. Asiat. Soc. 54: 33 (1910). - TYPE: [Peninsular Malaysia], Perak, Gopeng, July 1883, King's Collector 4673 (lectotype CAL, designated by Kanis, Blumea 16: 46 (1968); isolectotypes BM [BM000798711], BO, K [K001129818], L [L0012447], P [P05467121], SING [SING0054186, SING0054187, SING0054188]).

Brackenridgea serrulata Bartell., Malpighia 15: 163, t. 9 (1901). - TYPE: Borneo, Danao Lamadigian, May 1867, Beccari P.B. 3469 (holotype FI [FI007764]; isotypes K [K000657988], L [L0012451]).

Brackenridgea kingii Tiegh., Ann. Sci. Nat., Bot. sér. 8, 16: 395 (1902), as 'Kingi'. - TYPE: [Peninsular Malaysia], Perak, Larut, July 1884, King's Collector 6396 (lectotype P [P01817424], designated by Kanis, Blumea 16: 46 (1968); isolectotypes BM [BM000798712], K [K000501142], L [L0012446], US [US00169547]).

Brackenridgea rubescens Tiegh., Ann. Sci. Nat., Bot. sér. 8, 16: 396 (1902). TYPE: Singapore, Changi, [1890], Ridley 2072 [leg. J.S. Goodenough] (lectotype P [P01817425], designated by Kanis, Blumea 16: 46 (1968); isolectotypes CAL, K [K001129819], L [L0012448], SING [SING0054237]). 
2. Campylospermum Tiegh. in Morot, J. Bot. 16: 35 (1902). - Ochna sect. Campylospermum (Tiegh.) Kuntze, Lex. Gen. Phan. 394 (1903). - TYPE: non desig.

Notes. The circumscription and nomenclature of the genera of Ochnaceae subtribe Ourateinae (sensu Kanis 1968: 15) continue to cause problems. Taxonomic treatments have varied between a single genus: Ouratea Aubl. (Gilg, 1925; Robson, 1963), to the 34 genera recognised by Van Tieghem (1907). There is general agreement now that Ouratea is an entirely New World genus (Farron, 1968; Amaral \& Bittrich, 2014; Schneider et al., 2014). The bulk of the Old World species were recognised in Gomphia Schreb. by Kanis (1968). Gomphia is actually a synonym of Ouratea, so Campylospermum Tiegh. was considered the correct generic name for the Old World species (Bittrich \& Amaral, 1994). Recently, Greuter \& Rankin Rodríguez (2014) analysed the typification of Gomphia once more and corrected the lectotype yet again, but this did not change the position of the generic name as a synonym of Ouratea. They pointed out that there is a generic name, Philomeda Thouars, older than Campylospermum, which under the rules of priority should be taken up for the African and Asian species. There is also the overlooked Valkera Stokes, which is not an orthographic variant but a deliberate renaming of Walkera Schreb. About 55 species from Africa, Madagascar and Asia are currently recognised in Campylospermum. In order to avoid further name changes, a proposal to conserve Campylospermum against Philomeda (for which no valid names at species rank have ever been made) and other earlier generic names might be made. However, there is growing evidence that Campylospermum is polyphyletic, with the West and Central African species representing a different genus to the East African, Madagascan and Asian species (Schneider et al., 2021; Shah et al., in press). Given the current state of flux, here I continue with the use of Campylospermum as the correct generic name for the present.

2.1 Campylospermum serratum (Gaertn.) Bittrich \& M.C.E.Amaral, Taxon 43: 92 (1994). - Meesia serrata Gaertn., Fruct. Sem. P1. 1: 344, t. 70, fig. 6 (1788). - Walkera serrata (Gaertn.) Willd., Sp. Pl., ed. 4, 1: 1145 (1798). - Valkera serrata (Gaertn.) Stokes, Bot. Mat. Med. 1: 447 (1812). - Ouratea serrata (Gaertn.) N.Robson, Taxon 11: 50 (1962). - Gomphia serrata (Gaertn.) Kanis, Taxon 16: 422 (1967). - TYPE: Ceylon, Koenig s.n. (lectotype L [L0144816] ex Carpologica Herb. Lugd. Bat., no. 1009, designated by Kanis, Blumea 16: 53 (1968)).

Gomphia angustifolia Vahl, Symb. Bot. 2: 49 (1791). - Ouratea angustifolia (Vahl) Baill. ex Laness., Pl. Util. Col. Franc. 607 (1886). - Ochna angustifolia (Vahl) Kuntze, Revis. Gen. Pl. 1: 106 (1891). - Campylospermum angustifolium (Vahl) Tiegh. in Morot, J. Bot. 16: 197 (1902). - TYPE: Zeilon [Sri Lanka], Koenig s.n. (holotype C [C10016040]; isotype P [P01817448]).

Ochna zeylanica Lam., Encycl. 4: 512 (1797). - Gomphia zeylanica (Lam.) DC., Ann. Mus. Natl. Hist. Nat. 17: 415 (1811). - Campylospermum zeylanicum (Lam.) 
Tiegh., Bull. Mus. Hist. Nat. (Paris) 9: 77 (1903). - Ouratea zeylanica (Lam.) Alston, Ann. Roy. Bot. Gard. (Peradeniya) 11: 209 (1929). - TYPE: [Published illustration] 'JABOTAPITA Cinnamomi folio, floribus spicatis. Nobis' in Burman, Thes. Zeylan. t. 56 (1736) (lectotype designated here).

Gomphia malabarica DC., Ann. Mus. Natl. Hist. Nat. 17: 416 (1811). Campylospermum malabaricum (DC.) Tiegh., Bull. Mus. Hist. Nat. (Paris) 9: 77 (1903). - TYPE: [Published illustration] 'Poea-tsjetti' in Rheede, Hort. Malab. 5: t. 52 (1685) (lectotype designated here).

Gomphia sumatrana Jack, Malayan Misc. 1(5): 29 (1820). - Ochna sumatrana (Jack) Kuntze, Revis. Gen. P1. 1: 106 (1891). - Ouratea sumatrana (Jack) Gilg in Engler \& Prantl, Nat. Pflanzenfam. 3(6): 142 (1893). - Campylospermum sumatranum (Jack) Tiegh. in Morot, J. Bot. 16: 197 (1902). - TYPE: Sumatra, Marsden s.n. (neotype K [K000657995], designated here).

Ochna crocea Griff., Not. P1. Asiat. 4: 463 (1854). - Ouratea crocea (Griff.) Burkill, Bull. Misc. Inform. Kew 1935: 318 (1935). - TYPE: Mergui, ad littoram maria Ins. Madamacca Pator: 1835, fructifer tant (not traced).

Ouratea borneensis Bartell., Malpighia 15: 156, t. 6 (1901). - Campylospermum borneense (Bartell.) Tiegh. in Morot, J. Bot. 16: 197 (1902). - TYPE: Borneo, Sarawak, Marop, March 1867, Beccari P.B. 3138 [cited as 318] (lectotype FI [FI007759], designated by Kanis, Blumea 16: 54 (1968); isolectotypes G [G00383852], K [K000657993], M [M-0213018], P [P01817460]).

Ouratea neriifolia Bartell., Malpighia 15: 158, t. 7 (1901), as 'neerifolia'. Campylospermum neriifolium (Bartell.) Tiegh., Ann. Sci. Nat., Bot. sér. 8, 16: 304 (1902). - TYPE: Borneo, Sarawak, Tg. Datu, April 1867, Beccari P.B. 3547 (holotype FI [FI007761]).

Ouratea beccariana Bartell., Malpighia 15: 159, t. 9 (1901). - Campylospermum beccarianum (Bartell.) Tiegh., Ann. Sci. Nat., Bot. sér. 8, 16: 301 (1902). - TYPE: Borneo, Sarawak, Bintulu, September 1867, Beccari P.B. 4025 (holotype FI [FI007758]; isotype K [K000657992]).

Cercinia thorelii Tiegh. in Morot, J. Bot. 16: 198 (1902), as 'Thoreli'. - Ouratea thorelii (Tiegh.) Lecomte, Fl. Indo-Chine 1: 704 (1911). - TYPE: Cochinchina, between Saigon and Bien-hoa, 1862-1866, Thorel 643 (lectotype P [P01817437], first step designated by Kanis, Blumea 16: 54 (1968), second step designated here; isolectotypes C, CAL, E, G [G00383822, G00383829, G00383849], P [P01817438]).

Campylocercum striatum Tiegh., Ann. Sci. Nat., Bot. sér. 8, 16: 304 (1902). - Ouratea striata (Tiegh.) Lecomte, Fl. Indo-Chine 1: 703 (1911), nom. illeg. non (Tiegh.) Urb. 
(1908). - Gomphia striata (Tiegh.) C.F.Wei, Fl. Reipubl. Popularis Sin. 49(2): 306 (1984). - Campylospermum striatum (Tiegh.) M.C.E.Amaral, Fl. China 12: 362 (2007). - TYPE: [Vietnam], Annam, Tourane, January 1837, Gaudichaud 291 (lectotype P [P01817419], first step designated by Kanis, Blumea 16: 54 (1968), second step designated here; isolectotypes P [P01817420, P01817421]).

Campylocercum zollingeri Tiegh., Ann. Sci. Nat., Bot. sér. 8, 16: 305 (1902). - TYPE: [Sumatra, Lampongs, between Tarabangie and Henyala, 20-21 September 1845], Zollinger 3075 (lectotype P [P01817461], first step designated by Kanis, Blumea 16: 54 (1968), second step designated here; isolectotypes BM, BO, G [G00340886, G00340887, G00340893], L [L.2391256], P [P01817462, P01817463]).

Cercinia brevis Tiegh., Ann. Sci. Nat., Bot. sér. 8, 16:310 (1902). - TYPE: Laos, Bassin de Sé-Moun, Mt S. Treng, December 1875, Harmand 131 (lectotype P [P01817413], first step designated by Kanis, Blumea 16: 54 (1968), second step designated here; isolectotypes BM, BR [BR000000521996], E, G [G00383805], K, P [P01817414, P01817415]).

Campylocercum hohenackeri Tiegh., Bull. Mus. Hist. Nat. (Paris) 9: 86 (1903). TYPE: India, Canara, Mangalore, 1849, Hohenacker 89 (lectotype P [P01817447], first step designated by Kanis, Blumea 16: 55 (1968), second step designated here; isolectotypes P [P01817441, P01817443, P01817445]).

Campylocercum metzii Tiegh., Bull. Mus. Hist. Nat. (Paris) 9: 86 (1903), as 'Metzi'. TYPE: India, 1858, Metz 2225 (lectotype P [P01817450], first step designated by Kanis, Blumea 16: 55 (1968), second step designated here; isolectotypes G [G00340892], P [P01817451]).

Campylocercum paucifolium Tiegh., Bull. Mus. Hist. Nat. (Paris) 9: 86 (1903). TYPE: India, Canara, Mangalore, 1849, Hohenacker 89 (lectotype P [P01817440], first step designated by Kanis, Blumea 16: 55 (1968), second step designated here; isolectotypes P [P01817442, P01817444]).

Campylospermum abbreviatum Tiegh., Bull. Mus. Hist. Nat. (Paris) 9: 80 (1903). TYPE: Peninsular Malaysia, Perak, Larut, September 1884, King's Collector 6598 (lectotype P [P01817464], designated by Kanis, Blumea 16: 55 (1968); isolectotype US [US00169552]).

Campylospermum cumingii Tiegh., Bull. Mus. Hist. Nat. (Paris) 9: 80 (1903). TYPE: Philippines, Luzon, Manila, Cuming 1520 (lectotype P [P01817457], first step designated by Kanis, Blumea 16: 55 (1968), second step designated here; isolectotypes G [G00340882, G00340883, G00340888], M [M-0213075], MEL [MEL2422054], P [P01817458]). 
Campylospermum kingii Tiegh., Bull. Mus. Hist. Nat. (Paris) 9: 79 (1903), as 'Kingi'. - TYPE: Peninsular Malaysia, Perak, Larut, September 1882, King's Collector 3370 (lectotype P [P01817465], designated by Kanis, Blumea 16: 55 (1968); isolectotypes G [G00340889], K [K001129821], L [L.2391399], US [US00169550]).

Campylospermum leschenaultii Tiegh., Bull. Mus. Hist. Nat. (Paris) 9: 76 (1903), as 'Leschenaulti'. - TYPE: Ceylon, Leschenault s.n. (holotype P [P01817453]).

Campylospermum nodosum Tiegh., Bull. Mus. Hist. Nat. (Paris) 9: 77 (1903). - TYPE: Ceylon, 1854, Thwaites C.P. 2412 (holotype P (not traced)).

Campylospermum perakense Tiegh., Bull. Mus. Hist. Nat. (Paris) 9: 80 (1903). Gomphia oblongifolia Ridl., Bull. Misc. Inform. Kew 1925: 281 (1925), nom. illeg. superfl. - TYPE: Peninsular Malaysia, Perak, Gopeng, Kinta, July 1883, King's Collector 4545 (lectotype P [P01817466], first step designated by Kanis, Blumea 16: 55 (1968), second step designated here; isolectotypes G, K [K001129808], L [L.2391398], P [P01817467]).

Campylospermum plicatum Tiegh., Bull. Mus. Hist. Nat. (Paris) 9: 78 (1903). - TYPE: Détroit de Malacca, Ile de Singapoor, Voyage de l'Astrobale et de la Zélée, 1838-1840, Hombron s.n. (lectotype P [P01817468], first step designated by Kanis, Blumea 16: 55 (1968), second step designated here; isolectotypes A, P [P01817469, P01817470]).

Campylospermum retinerve Tiegh., Bull. Mus. Hist. Nat. (Paris) 9: 75 (1903). - TYPE: India, 'Gurunda Karpara', Hb Vaillant s.n. (holotype P [P00660016]).

Campylospermum rheedei Tiegh., Bull. Mus. Hist. Nat. (Paris) 9: 78 (1903), as 'Rheedi'. - TYPE: [Published illustration] 'Tsjocatti', Rheede, Hort. Malab. 5: t. 48 (1685) (lectotype designated here).

Campylospermum strictum Tiegh., Bull. Mus. Hist. Nat. (Paris) 9: 79 (1903). TYPE: Peninsular Malaysia, Malacca, Batu Tiga, 1892, Derry 987 (lectotype P [P01817471], designated by Kanis, Blumea 16: 55 (1968); isolectotypes CAL, SING [SING0117113]).

Campylospermum thwaitesii Tiegh., Bull. Mus. Hist. Nat. (Paris) 9: 77 (1903). - TYPE: Ceylon, 1854, Thwaites C.P. 2412 (lectotype P [P01817454], first step designated by Kanis, Blumea 16: 55 (1968), second step designated here; isolectotype $\mathrm{P}[\mathrm{P} 01817455])$.

Campylospermum vahlianum Tiegh., Bull. Mus. Hist. Nat. (Paris) 9: 76 (1903). TYPE: India, Breyn s.n. (holotype P [P01817452]). 
Campylospermum walkeri Tiegh., Bull. Mus. Hist. Nat. (Paris) 9: 76 (1903), as 'Walkeri'. - TYPE: Ceylon, 1837, Walker 54 (holotype P [P01817457]).

Campylospermum wallichianum Tiegh., Bull. Mus. Hist. Nat. (Paris) 9: 76 (1903). - TYPE: [Locality unknown], Wallich s.n. [EIC 2803] (lectotype P [P01817471], designated by Kanis, Blumea 16: 55 (1968); possible isolectotypes A, BM, E, G [G00383863, G00383868], K, K-W [K001117120, K001117121]).

Cercinia doumeri Tiegh., Bull. Mus. Hist. Nat. (Paris) 9: 87 (1903). - TYPE: India, 1821, Doumer s.n. (holotype P [P01817449]).

Cercinia wightii Tiegh., Bull. Mus. Hist. Nat. (Paris) 9: 87 (1903), as 'Wighti'. - TYPE: Ceylon, March 1836, Wight s.n. [Peninsula Indiae Orientalis No. 396] (holotype P [P01817456]).

Cercinia annamensis Tiegh., Ann. Sci. Nat., Bot. sér. 9, 5: 163, 184 (1907). - TYPE: Annam, Hué, 1877, Harmand s.n. [Hb Pierre 7027] (holotype P [P01817422]).

Cercinia elongata Tiegh., Ann. Sci. Nat., Bot. sér. 9, 5: 163, 184 (1907). - TYPE: Cochinchina, 1876-1877, Harmand 144 [Hb Pierre 7026] (holotype P [P01817439]).

Gomphia microphylla Ridl., Fl. Malay Penins. 1: 365, fig. 38 (1922). - Ouratea microphylla (Ridl.) Craib, Fl. Siam. 1: 245 (1926). - TYPE: Peninsular Malaysia, Langkawi, Pulau Dayang Bunting, 7 December 1916, Robinson 6197 (lectotype K [K000658000], designated by Kanis, Blumea 16: 56 (1968); isolectotype K [K000658000]).

Ouratea arcta Craib, Bull. Misc. Inform. Kew 1926: 341 (1926). - TYPE: Thailand, Kaw Chang, Klawng Mayum, 3 April 1923, Kerr 6849 (lectotype K [K000657998], designated by Kanis, Blumea 16: 56 (1968); isolectotypes BM [BM000798578, BM000798579], SING [SING0294228], TCD [TCD0013539]).

Ouratea megacarpa Ridl., Bull. Misc. Inform. Kew 1930: 76 (1930). - TYPE: Borneo, Sarawak, Mt Matang, January 1915, Ridley s.n. (holotype K [K000657994]).

Ouratea sumatrana (Jack) Gilg var. nervosa Craib, Fl. Siam. 1: 245 (1926). - TYPE: Thailand, Phuket, Kopah, 8 December 1917, Mhd Haniff \& Mhd Nur SFN 2074 (lectotype K [K001129809], designated by Kanis, Blumea 16: 56 (1968); isolectotypes BM, BO, BRI, SING [SING0054190]).

Notes. Kanis (1968) recognised a single species for what he called Gomphia in Asia. His Gomphia serrata occurs from India and Sri Lanka to the Philippines and includes numerous synonyms, mostly species described by Van Tieghem. Perusal of herbarium material shows substantial variation in form within the species. Recently, 
Amaral (Zhang \& Amaral, 2007) reinstated one of the synonyms as Campylospermum striatum, a species from Hainan and Vietnam. For the present, I prefer to maintain the broad view espoused by Kanis, but note that a thorough revision of the complex is needed.

3. Euthemis Jack, Malayan Misc. 1(5): 15 (1820). - TYPE: Euthemis leucocarpa Jack (lectotype designated by Pfeiffer, Nomencl. Bot. 1(2): 1316 (1873)).

3.1 Euthemis leucocarpa Jack, Malayan Misc. 1(5): 16 (1820). - TYPE: Singapore, 1822, Wallich s.n. [EIC 2516 p.p.] (neotype K-W [K001116327], first step designated by Kanis, Blumea 16: 63, 65 (1968), second step designated here; isoneotypes K [K000657983], K-W [K001116329]).

Euthemis robusta Hook.f., Trans. Linn. Soc. London 23: 163 (1860). - TYPE: Borneo, 1853, Lobb s.n. (lectotype K [K000657982], first step designated by Kanis, Blumea 16: 63 (1968), second step designated here; isolectotypes K [K000657981], P [P01817430]).

Euthemis leucocarpa Jack var. latifolia Miq., Fl. Ned. Ind., Eerste Bijv. 3: 533 (1861). - TYPE: Banka, Plangas, Teijsmann H.B. 3375 (lectotype L [L0012455], first step designated by Kanis, Blumea 16: 63 (1968), second step designated here; isolectotypes BO, L [L0012454], P [P01817431], U).

Notes. Kanis (1968: 65) wrote 'The Kew specimen that is marked as the type of this species, is certainly a duplicate from the collection Wallich 2516 p.p. made at Singapore in 1822. I accept this collection as neotype of E. leucocarpa Jack'. The Wallich Catalogue number 2516 includes two gatherings, one made by Wallich in Singapore in 1822, and another from Penang in 1823, probably by George Porter. These are mounted rather haphazardly across five sheets in $\mathrm{K}-\mathrm{W}$ with various original labels but no indication of which material belongs to which collection except on a couple of smaller sheets Wallich has written the Singapore collection details directly on the sheet. As Kanis was clearly referring to more than one specimen, a secondstep typification is proposed here to restrict the choice of neotype to the better of the small sheets. The specimen [K000657983] in a type cover in the K general herbarium mentioned by Kanis is dated October 1823 on Wallich's label, possibly indicating some confusion with the Penang gathering.

3.2 Euthemis minor Jack, Malayan Misc. 1(5): 18 (1820). - TYPE: Singapore, 1822, Wallich s.n. [EIC 2517] (neotype K-W [K001116332], first step designated by Kanis, Blumea 16: 65 (1968), second step designated here; isoneotype K-W [K001116333]). 
Euthemis obtusifolia Hook.f., Trans. Linn. Soc. London 23: 163 (1860). - TYPE: Borneo, 1853, Lobb s.n., (holotype K [K000657979]).

Euthemis engleri Gilg in Engler \& Prantl, Nat. Pflanzenfam. 3(6): 152, 153, fig. 78 (1893). - TYPE: [Published illustration] Gilg in Engler \& Prantl, Nat. Pflanzenfam. 3(6): 152, fig. 78 (1893) (lectotype designated here).

Euthemis ciliata H.Pearson, Bull. Misc. Inform. Kew 1906: 3 (1906). - TYPE: Sumatra, Siak, Penassa, November 1897, Ridley 9000 (holotype K [K000657980, K001129816 - i.e. two sheets comprising a single specimen]; isotypes P, SING [SING0054193]).

Euthemis hackenbergii Diels, Bot. Jahrb. Syst. 60: 310 (1926). - TYPE: S. Borneo, Sampit, 11 June 1923, Hackenberg 10 (B, destroyed).

ACKNOWLEDGEMENTS. Roxali Bijmoer (L) very kindly located the lectotype of Meesia serrata and forwarded photographs.

\section{References}

Amaral, M.C.E. \& Bittrich, V. (2014). Ochnaceae. In: Kubitzki, K. (ed.) The Families and Genera of Vascular Plants, vol. 11, pp. 253-268. Berlin: Springer-Verlag.

Bittrich, V. \& Amaral, M.C.E. (1994). Lectotypification of Gomphia Schreb. Taxon 43: 89-93.

Farron, C. (1968). Contribution à la taxonomie des Ourateeae (Ochnaceae) d'Afrique. I. Partie générale. Candollea 23: 177-228.

Gilg, E. (1925). Ochnaceae. In: Engler, A. \& Prantl, K. (eds) Die Natürlichen Pflanzenfamilien, ed. 2, vol. 21, pp. 53-87. Leipzig: W. Engelmann.

Govaerts, R. (1996). World Checklist of Seed Plants, vol. 2, pt. 1. Antwerp: Continental Publishing.

Greuter, W. \& Rankin Rodríguez, R. (2014). A type for Gomphia (Ochnaceae)—once again. Taxon 63: 1122-1123.

Ho, B.C., Lua, H.K., Bazilah Ibrahim, Yeo, R.S.W., Athen, P., Leong, P.K.F., Ali Ibrahim, Koh, S.L., Hassan Ibrahim, Lindsay, S., Chin, L.L., Seah, W.W. \& Middleton, D.J. (2019). The plant diversity of Bukit Timah Nature Reserve, Singapore. Gard. Bull. Singapore 71(Suppl. 1): 41-134.

Kanis, A. (1968). A revision of the Ochnaceae of the Indo-Pacific Area. Blumea 16: 1-82.

Lendemer, J.C. (2020). Epitypes are forever: best practices for an increasingly misused nomenclatural action. Taxon 69: 849-850.

Robson, N.B.K. (1963). Ochnaceae. In: Exell, A.W., Fernandes, A. \& Wild, H. (eds) Flora Zambesiaca, vol. 2, pt. 1, pp. 224-262. London: Royal Botanic Gardens, Kew.

Schneider, J.V., Bissiengou, P., Amaral, M.C.E., Ali Tahir, Fay, M.F., Thines, M., Sosef, M.S.M., Zizka, G. \& Chatrou, L.W. (2014). Phylogenetics, ancestral state reconstruction, and a new infrafamilial classification of the pantropical Ochnaceae (Medusagynaceae, Ochnaceae s.str., Quiinaceae) based on five DNA regions. Molec. Phylogen. Evol. 78: 199-214. 
Schneider, J.V., Jungcurt, T., Cardoso, D., Amorim, A.M., Töpel, M., Andermann, T., Poncy, O., Berberich, T. \& Zizka, G. (2021). Phylogenomics of the tropical plant family Ochnaceae using targeted enrichment of nuclear genes and 250+ taxa. Taxon 70: 48-71.

Shah, T., Schneider, J.V., Zizka, G., Maurin, O., Baker, W., Forest, F., Brewer G.E., Savolainen, V., Darbyshire, I. \& Larridon, I. (In press). Joining forces in Ochnaceae phylogenomics: a tale of two targeted sequencing probe kits. Amer. J. Bot.

Van Tieghem, P. (1907). Supplément aux Ochnacées. Ann. Sci. Nat., Bot., sér. 9, 5: 157-192.

Wong, H.F., Tan, S.Y., Koh, C.Y., Siow, H.J.M., Li, T., Heyzer, A., Ang, H.F., Mirza Rifqi bin Ismail, Srivathsan, A. \& Tan, H.T.W. (2013). Checklist of the Plant Species of Nee Soon Swamp Forest, Singapore: Bryophytes to Angiosperms. Singapore: National Parks Board and Raffles Museum of Biodiversity Research, National University of Singapore.

Zhang, Z. \& Amaral, M.C.E. (2007). Ochnaceae. In: Wu, Z.Y. \& Raven, P. (eds) Flora of China, vol. 12, pp. 361-363. Beijing: Science Press; and St. Louis: Missouri Botanical Garden Press. 
